\title{
Velocity spectrum imaging using radial k-t SPIRiT
}

\author{
Claudio Santelli ${ }^{1,2^{*}}$, Sebastian Kozerke ${ }^{2,1}$, Tobias Schaeffter ${ }^{1}$ \\ From 15th Annual SCMR Scientific Sessions \\ Orlando, FL, USA. 2-5 February 2012
}

\section{Background}

Fourier velocity encoding (FVE) [P.R.Moran,MRI $(1), 1982$ ] assesses the distribution of velocities within a voxel by acquiring a range of velocity encodes $\left(\mathrm{k}_{\mathrm{v}}\right)$ points. The ability to measure intra-voxel phase dispersion, however, comes at the expense of clinically infeasible scan times. We have recently extended [C.Santelli, $\operatorname{ESMRMB}(345), 2011]$ the auto-calibrating parallel imaging technique SPIRiT [M.Lustig,MRM(64),2010] to exploit temporal correlations in dynamic k-t signal space and successfully applied it to radially undersampled FVE data. Prior assumption of Gaussian velocity spectra additionally allows undersampling along the velocity encoding dimensions [P.Dyverfeldt,MRM $(56), 2006]$. In this work, a scheme is proposed to nonuniformly undersample the $\mathrm{k}_{\mathrm{v}}$-axes in addition to undersampling $\mathrm{k}-\mathrm{t}$ space for reconstructing mean and standard deviation (SD) of the velocity spectra for each voxel in aortic flow measurements.

\section{Methods}

\section{Acquisition}

$2 \mathrm{D}$ radial $(\mathrm{FOV}=250 \mathrm{~mm} \times 250 \mathrm{~mm})$ fully sampled cine FVE data of the aortic arch for 3 orthogonal velocity components was obtained from 5 healthy volunteers on a 3T Philips Achieva scanner (Philips Healthcare, Best, The Netherlands) using a 6 element receive array. Three different first gradient moments corresponding to encoding velocities of $25 \mathrm{~cm} / \mathrm{s}, 50 \mathrm{~cm} / \mathrm{s}$ and $200 \mathrm{~cm} / \mathrm{s}$ were applied along with a reference point $\left(\mathrm{k}_{\mathrm{v}}=0\right)$. Undersampled radial data sets were obtained by separately regridding these 4-point measurements onto Golden-angle profiles (Fig.1a).
'Division of Biomedical Engineering and Imaging Sciences, King's College London, London, UK

Full list of author information is available at the end of the article

\section{Reconstruction}

The interpolation operator G, enforcing consistency between calibration data from a fully sampled centre of $\mathrm{k}$-space and reconstructed Cartesian k-space points, $\mathbf{x}$, is extended for dynamic MRI by including temporal correlations between adjacent data frames (Fig.1b). Data consistency is imposed using gridding-operator D (Fig.1a). Then, $\mathbf{x}$ is recovered by solving the minimization problem in Fig.1d). Reconstruction was performed for every $\mathrm{k}_{\mathrm{v}}$-point separately using dedicated software implemented in Matlab (Natick,MA,USA). A 7x7x3 neighborhood in $k_{x}-k_{y}-t$ space was chosen for the $k-t$ space interpolation kernel. The weights were calculated from a 30x30x ( $\mathrm{nr}$ cardiac phases) calibration area (Fig.1c). Mean and SD of velocity distributions were calculated for the resulting coil-combined images.

\section{Results}

Fig.2a) compares the mean root-mean-square error (RMSE) of the reconstructed mean velocities and SDs in the aortic arch for different undersampling factors and for each flow direction (M-P-S). Fig.2b) shows in-plane streamlines reconstructed from the acquired mean velocities and turbulence intensity maps calculated from SD values.

\section{Conclusions}

A novel auto-calibrating reconstruction technique for dynamic radial imaging was successfully applied to undersampled 4-point FVE data from five healthy volunteers. Results show that up to 12 -fold radial undersampling provides accurate quantification of mean velocities and turbulence intensities derived from velocity spectra.

\footnotetext{
Author details

'Division of Biomedical Engineering and Imaging Sciences, King's College London, London, UK. ${ }^{2}$ Institute for Biomedical Engineering, University and ETH Zurich, Zurich, Switzerland.
}

Published: 1 February 2012 


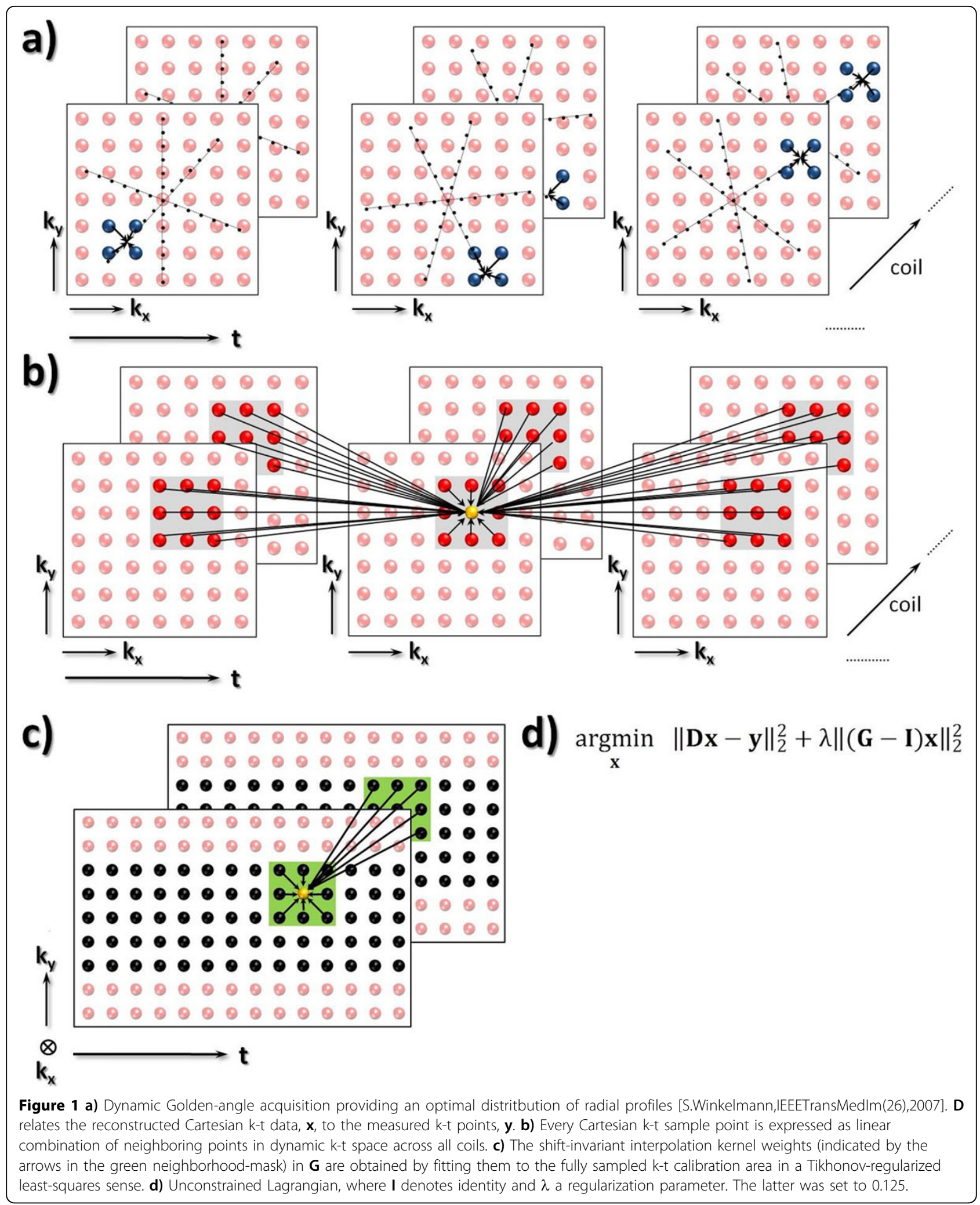




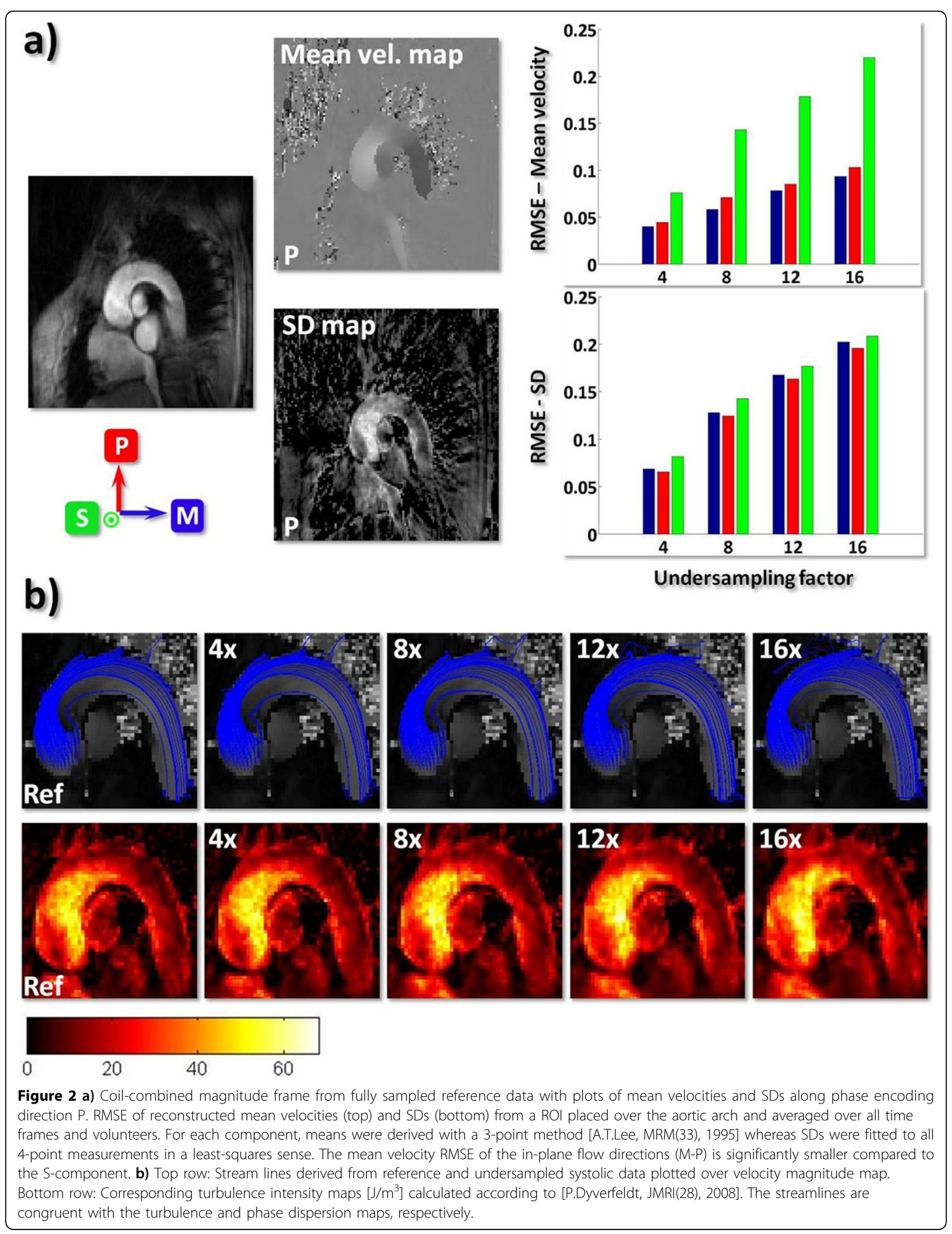


doi:10.1186/1532-429X-14-S1-W59

Cite this article as: Santelli et al:: Velocity spectrum imaging using radial

k-t SPIRiT. Journal of Cardiovascular Magnetic Resonance 2012 14(Suppl 1):

W59.

Submit your next manuscript to BioMed Central and take full advantage of:

- Convenient online submission

- Thorough peer review

- No space constraints or color figure charges

- Immediate publication on acceptance

- Inclusion in PubMed, CAS, Scopus and Google Scholar

- Research which is freely available for redistribution

Submit your manuscript at 